\title{
Causes and prevention of cherry cracking: a review
}

\begin{abstract}
Cherry fruit is considered to be quite sensitive to cracking and is the major limiting factor for profitable cherry production in most of the cherry-producing regions of the world. At times, the cracking severity can reach up to $90 \%$. Although most of the fruit cracking is attributed to direct and possibly localized water uptake through the fruit skin, there are many other interactive factors that can contribute to cracking including the variety grown, skin properties, climate, and so on. The cracking in cherry has been categorized in three distinct forms: stem end cuticular fractures, nose or apical cracking, and the third, side cracking, which is a large crack usually deep into the pulp on the cheek of the fruit and is considered to be most damaging. The type of the crack developed may depend on the particular etiological factor and the shape of the fruit. However, the problem can be minimized to a great extent by knowing the cause and accordingly, adapting certain management practices, of which irrigation management, protective covers, and mineral supplements are of prime consideration.
\end{abstract}

Keyword: Cherry cracking; Rain; Water uptake; Protectants 